\title{
TROUBLES ET TENSIONS INTERNES: POUR UNE NOUVELLE APPROCHE HUMANITAIRE?
}

La violence a coutume

d'engendrer la violence

Eschyle

Ce que l'on appelle généralement «troubles intérieurs» et «tensions internes» (ou «troubles et tensions internes») recouvre un ensemble de situations qui se caractérisent toutes par des phénomènes de violence liés à des violations des droits de l'homme entraînant des souffrances humaines. Ces situations, actuellement fréquentes et répandues dans le monde, sont caractérisées par des affrontements directs entre les forces de l'ordre et des opposants au régime ou par toute une gamme de tensions, de caractère ethnique ou racial, religieux ou idéologique, économique ou social, lesquelles peuvent aussi déterminer l'intervention des forces de l'ordre à titre préventif.

Mais au-delà de ces phénomènes dont la description est volontairement simplifiée, se profile le spectre de la violence, publique ou clandestine, avec son cortège d'abus et d'infractions à des droits de l'homme fondamentaux: arrestations massives, mauvais traitement de détenus, mesures excessives de répression, disparition de personnes, actes de terrorisme, exécutions sommaires, etc.

Les effets de ces situations de violence à l'intérieur des Etats peuvent être, selon les cas, d'ordre politique ou juridique, économique ou social; ils sont par contre toujours d'ordre humanitaire dans la mesure où ils affectent, à plus ou moins grande échelle, l'individu dans sa vie, dans son intégrité, dans sa dignité. 
Certes, les violations des droits de l'homme sont un sujet constant de préoccupation pour l'Organisation des Nations Unies; certes, le CICR ainsi que d'autres organismes n'ont pas ménagé leurs efforts pour soulager les souffrances des victimes. Mais les résultats n'ont pas répondu pleinement aux espérances: sans vouloir comme certains experts parler de «vide juridique» à propos des droits et obligations applicables dans ces situations, force est de reconnaitre que la protection internationale des victimes des troubles et tensions internes reste insuffisante.

En fait, la question fondamentale est la suivante: comment mieux protéger l'individu contre la violence dans tous ses aspects? Si la communauté internationale a acquis un capital juridique important dans le domaine des droits de l'homme et du droit humanitaire, le temps est-il venu de rappeler et de réaffirmer quelques règles fondamentales qui doivent être observées même dans les situations de troubles et tensions internes? Ou convient-il de garder une approche pragmatique et nonjuridique vis-à-vis des gouvernements et de ces situations?

$* * *$

La communauté internationale dispose d'instruments juridiques internationaux susceptibles de s'appliquer à de telles situations. En premier lieu, la législation universelle et régionale en matière de droits de l'homme définit les droits de l'individu dont les Etats s'engagent à garantir le respect et la mise en auvre. Certes, en cas de troubles, les Etats peuvent suspendre l'exercice de certains de ces droits, mais même s'ils ont décrété l'état d'exception, ils restent tenus de préserver en tout temps et en toutes circonstances un minimum de droits fondamentaux nécessaires pour assurer la sauvegarde de l'intégrité physique et morale de l'individu.

En vérité, bien que formellement applicable, tant en période de paix que de conflit, le droit des droits de l'homme est essentiellement conçu pour le temps de paix. L'exercice de ces droits se trouve dans la pratique très souvent réduit lors de confrontations, sans compter que l'état de ratification des instruments internationaux et régionaux des droits de l'homme reste très insuffisant.

Le droit international humanitaire assure une protection de l'individu non seulement contre l'ennemi étranger, mais aussi dans certaines circonstances contre son propre gouvernement. Ce progrès trouve son expression la plus claire dans l'article 3 commun aux quatre Conventions de Genève qui contient en effet "un noyau de droits fondamentaux» que la doctrine considère comme normes minimales applicables à tout conflit 
armé et qui doivent être impérativement respectées. Leur contenu rejoint d'ailleurs, pour une bonne part, les normes non dérogeables des droits de l'homme, lesquelles doivent être respectées en toutes circonstances, même dans les situations de troubles et tensions internes.

Dans ces divers types de situations, le Comité international de la Croix-Rouge peut offrir ses services tant aux parties à un conflit non-international, en vertu de l'article 3, qu'à un gouvernement confronté à une situation de troubles et tensions internes, sur la base des Statuts du Mouvement international de la Croix-Rouge et du Croissant-Rouge, de ses propres statuts et de résolutions de la Conférence internationale de la Croix-Rouge et du Croissant-Rouge.

En outre, le droit d'initiative humanitaire général reconnu au CICR lui permet d'intervenir dans les situations de tensions internes, voire dans des cas qui n'auraient même pas été définis comme des tensions internes mais où l'action humanitaire d'une institution spécifiquement neutre et impartiale serait opportune.

Cette pratique du CICR d'offrir ses services est ancrée dans sa propre tradition. En se fondant sur ses nombreuses expériences concrètes, le CICR a qualifié ces situations sous l'angle humanitaire, il a cherché à en donner des définitions, mais bien que celles-ci n'engagent que lui, elles ont le mérite de mieux délimiter les bases de son action. Par ailleurs, il a aussi fixé un cadre à son action qui ne recouvre pas tous les aspects des troubles et tensions internes (notamment pendant les confrontations proprement dites) mais se concentre essentiellement sur les conséquences humanitaires de ces situations, spécialement à l'égard des détenus et de leurs familles.

L'amélioration des conditions de détention et du traitement des personnes détenues, les interventions spéciales à l'occasion de dénis de justice particulièrement flagrants, ou de violences indiscriminées contre des personnes sans défense, ou lors de prises d'otages, la lutte contre le phénomène des disparitions forcées, etc. Toutes ces activités de protection et d'assistance du CICR dans de telles situations non couvertes par le droit international humanitaire sont décrites et commentées dans le document de doctrine du CICR présenté ci-après, publié tout d'abord en 1986 à l'occasion de la XXVe Conférence internationale de la CroixRouge puis mis à jour à la lumière des nouveaux Statuts du Mouvement.

Il n'est pas dans notre propos d'évaluer ici l'action du CICR dans ces situations. Relevons que le droit d'initiative humanitaire dont jouit le CICR dans ces situations est devenu d'ordre coutumier et que ses offres de service ne peuvent être considérées comme une ingérence dans les affaires intérieures d'un Etat. Il n'en demeure pas moins que ce droit du 
CICR n'est pas assorti d'une obligation pour les Etats d'y répondre favorablement.

Constatant la prolifération des situations de troubles et tensions internes et l'aspect protéiforme de la violence qui les caractérise, des voix nombreuses se sont élevées pour déplorer les insuffisances des moyens juridiques visant à la protection des victimes. Et certains de regretter que les Protocoles additionnels aux Conventions de Genève aient exclu ces situations de leur champ d'application! D'autres pensent qu'en l'absence d'une obligation des Etats d'accepter les offres de service du CICR, même en temps de conflit armé non-international, une telle exclusion ne nuit pas à l'action humanitaire du Comité, au contraire peut-être. Preuve en est que le CICR a pu visiter des lieux de détention dans quelque 90 pays sans que ses interventions ne soient fondées sur une obligation des Etats.

Afin de répondre à ces préoccupations majeures, une nouvelle approche se fait jour qui consiste non à créer un nouveau droit spécifique de ces situations, mais plutôt à rappeler, pour mieux faire appliquer dans les situations de troubles et tensions internes, un ensemble de règles fondamentales existantes, faisant appel au droit écrit, au droit coutumier et aux principes généraux du droit, règles qui ne sauraient être transgressées sans porter atteinte à la conscience universelle.

La Revue est heureuse de présenter à ce propos deux textes qui répondent à cette approche, l'un est un «Code de conduite» proposé par un expert du CICR s'exprimant à titre personnel, l'autre un projet de "Déclaration type» émanant d'un internationaliste bien connu.

Leur démarche est identique dans la mesure où tous deux ont recours au «fond commun» du droit des droits de l'homme et du droit humanitaire et proposent des règles fondamentales qui doivent être respectées en toutes circonstances et sans discrimination.

Il n'est pas dans notre intention de procéder, dans le cadre de cet éditorial, à une analyse juridique comparative de ces deux textes. Le lecteur jugera. Nous nous bornerons à souligner ce qui en compose l'essence et ce qui en fait le prix: tous deux, que ce soit dans le préambule $d u$ Code de conduite ou à l'article 2 de la Déclaration, ont le souci majeur de rappeler des règles essentielles du droit international, écrit ou coutumier, qui s'adressent à tous, tout d'abord aux gouvernements chargés de maintenir ou rétablir l'ordre mais aussi à ceux qui s'opposent aux autorités. 
Ces règles qui doivent être impérativement respectées appartiennent aux domaines suivants: le droit à la vie, la dignité inhérente à la personne humaine; l'interdiction du meurtre, de la torture et autres traitements dégradants; la prise d'otages; les disparitions de personnes, les actes de terrorisme et les peines collectives; le recours à la force hors de proportion avec les buts pousuivis; un traitement humain aux personnes privées de liberté; l'octroi de garanties judiciaires fondamentales; les droits de l'enfant; la protection des blessés et des malades et la recherche des disparus.

On notera que ces règles impératives s'inspirent essentiellement de ce noyau de droits et interdictions non dérogeables mis notamment en relief dans le Pacte international relatif aux droits civils et politiques, la Convention américaine des droits de l'homme et la Convention européenne de sauvegarde des droits de l'homme ainsi que dans l'article 3 commun aux quatre Conventions de Genève et les articles 4 à 6 du Protocole II.

En outre les deux auteurs tiennent à préciser que les règles qu'ils présentent ne sauraient en aucune manière être interprétées comme une limitation de la protection accordée par le droit humanitaire et les instruments des droits de l'homme en vigueur.

En fait, ce qui distingue ces deux textes réside surtout dans la présentation et la formulation, lesquelles traduisent le souci de chaque auteur de trouver par là le moyen qui lui semble le plus adéquat pour convaincre les destinataires de ces textes et l'approche la mieux adaptée au contexte politique actuel.

Le Code de conduite se veut avant tout instrument de diffusion s'adressant à la conscience de chacun. Son langage simple et sa concision rappellent le recueil de "Règles fondamentales de droit international humanitaire applicables dans les conflits armés", élaboré en 1979 par des experts du CICR afin de faciliter la diffusion du droit humanitaire. Ce souci primordial de diffusion se traduit d'ailleurs dans la règle $13 \mathrm{du}$ Code de conduite.

La Déclaration type présente un caractère juridique plus accentué dans son exposition et sa formulation; elle est conçue comme un recueil de principes et règles impératifs assorti de dispositions procédurales visant à en expliciter et en faciliter l'application. En ce sens la Déclaration type prend le visage d'un Protocole additionnel aux Pactes des droits de l'homme et aux traités de droit humanitaire auxquels elle se réfère essentiellement. Et si l'auteur se montre discret quant à la nécessité de diffuser les règles contenues dans la Déclaration, il prend soin de veiller à ce que les autorités de l'Etat puissent «accorder aux organisations humanitaires toutes les facilités en leur pouvoir pour leur permettre 
d'assumer leurs tâches humanitaires afin d'apporter protection et assistance aux victimes des troubles intérieurs ou des tension internes».

Le Code de conduite évite la terminologie juridique afin d'être aussi accessible que possible à tous; il est fait pour inciter les individus à observer un certain comportement humanitaire et à ce titre il est avant tout dirigé vers les victimes.

La Déclaration type, de caractère juridique, s'adresse davantage aux Etats et aux citoyens, mais elle est suffisamment souple pour être à la fois un document de référence utilisable par tous et un projet susceptible d'être présenté à une instance internationale, universelle ou régionale.

Tels qu'ils sont, les deux textes sont autant de «ballons d'essai» qui ont déjà le mérite d'exister. Ils sont en quelque sorte des éléments prometteurs de cette nouvelle approche humanitaire que tous appellent de leurs væux dans un domaine particulièrement démuni.

En publiant ces deux textes, la Revue souhaite vivement amorcer une réflexion approfondie sur ce phénomène des troubles et tensions internes et sur les moyens d'en conjurer les effets sur le plan humanitaire. Elle invite ses lecteurs à se forger leur propre opinion et, notamment, à juger si, à ce stade, il conviendrait déjà de tenter de fixer la forme d'un instrument sur les troubles et tensions internes ou s'il est préférable d'en dégager l'esprit en s'adressant prioritairement à la conscience de tous, voire de renoncer à toute codification pour mettre l'accent sur la seule approche pragmatique de l'action humanitaire.

En tout état de cause, la Revue, en ouvrant ses colonnes à ses lecteurs, souhaite favoriser le dialogue entre tous ceux qui voudront bien lui apporter leurs commentaires et suggestions sur un sujet dont l'importance n'échappe à personne.

La Revue 